\title{
Study the Quality of Image Enhancement by using Retinex Technique which Capture by different Lighting (Sun and Tungsten)
}

\author{
Ali A. Al-Zuky \\ Professor, College of sciences, Al- \\ Mustansiryha Un. \\ Baghdad-Iraq
}

\author{
Salema S.Salman \\ College of pharmacy, Baghdad \\ Un. Baghdad-Iraq
}

\author{
Anwar H. Al-Saleh \\ College of sciences, Al- \\ Mustansiryha Un. \\ Baghdad-Iraq
}

\begin{abstract}
In this paper, the quality of the still image which is captured with difference sources of lighting (light of sun, light of tungsten) under different conditions was studying, thereby improving the resulting image of the imaging system using Retinex technique. Results were analyzed and estimated the quality improvement of capturing by using criterion of contrast depending of the point edge and by various statistical criteria based on the expense of mean, absolute of center moment and global contrast.
\end{abstract}

\section{Keywords}

Image enhancement, Retinex technique, Lighting.

\section{INTRODUCTION}

The visible portion of the electromagnetic spectrum extends from about 380 to about 780 nanometers is called light. The Illuminating Engineering Society of North America defines light as radiant energy that is capable of exciting the retina and producing a visual sensation. Light therefore, cannot be separately described in terms of radiant energy or of visual sensation but is a combination of them [1]. Radiometry is the study of optical radiation light, ultraviolet radiation, and infrared radiation. Photometry on the other hand is concerned with humans visual response to light Radiometry is concerned with the total energy content of the radiation, while photometry examines only the radiation that humans can see. Thus the most common unit in radiometry is the watt (W), which measures radiant flux (power), while the most common unit in photometry is the lumen $(\mathrm{lm})$, which measures luminous flux. For light at other wavelengths, the conversion between watts and lumens is slightly different, because the human eye responds differently to different wavelengths, radiant intensity is measured in watts/steroidal $(\mathrm{W} / \mathrm{sr})$, while luminous intensity is measured in candelas (cd, or $1 \mathrm{~m} / \mathrm{sr})[2,3]$. The human eye is more sensitive to some wavelengths than to others. This sensitivity depends on whether the eye is adapted for brightness or darkness because the human eye contains two types of photoreceptors cones and rods. When the eye is adapted for bright light, called photopic vision (luminance levels generally greater than about $3.0 \mathrm{~cd} / \mathrm{m}^{2}$ ), the cones dominate. At luminance levels below approximately 0.001 $\mathrm{cd} / \mathrm{m}^{2}$, the rods dominate in what is called scotopic vision. Between these two luminance levels, mesopic vision uses both rods and cones. Figure (1) shows the relative sensitivity to various wavelengths for cones (photopic) and rods (scotopic).

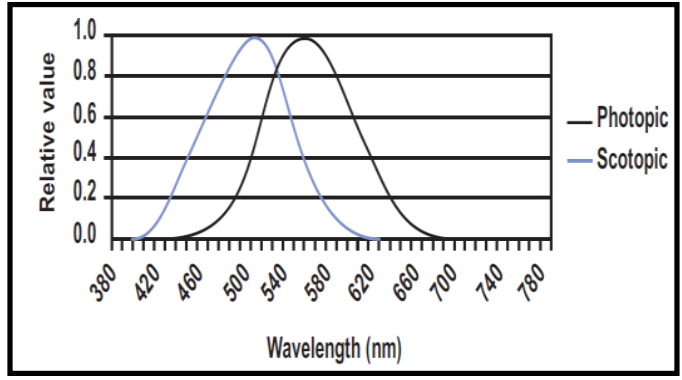

Fig 1: Relative Sensitivity function versus wavelength $[4,5]$

The curves represent the spectral luminous efficacy for human vision. The lumen is defined such that the peak of the photopic vision curve has a luminous efficacy of 683 lumens/watt. This value for the scotopic peak makes the efficacy the same as the photopic value at $555 \mathrm{~nm}$. The scotopic vision is primarily rod vision, and the photopic vision includes the cones. The previous work in color image processing techniques enhancement, will be given below:

- The researchers Zhixi Bian and Yan Zhang in 2002 study Retinex Image Enhancement Techniques Algorithm, Application and Advantages They will implement Single scale retinex(SSR), multiscale retinex(MSR), and color restoration method for MSR(MSRCR )with gain/offset. Where they adjust the gain/offset parameters to adjust most of the pixels values to display domain and clap small part of the values to improve the contrast. Comparisons with other image enhancement techniques will be made [6]

- Yaoyu cheng, Yu wang and Yan hu study in 2009 introduce image enhancement algorithm based on Retinex for Small-bore steel tube butt weld's X-ray imaging then determine the characteristics of X-ray images and the inadequacy of conventional enhancement methods then propose variable framework model of Retinex algorithm for the X-ray image enhancement, improve the detection efficiency and quality [7].

\section{RETINEX THEORY}

Color is important information source to describe, distinguish and identify an object for human and other biological visual system. In the image, the object can be displayed in different color saturation and has nothing to do with the change of the light. The human's visual perception is more sensitive to the reflection light of the object's surface [6, 7]. Retinex theory is introduced by Land to explain human's visual model, and establish illumination invariance model of 
which the color has nothing to do with. The basic objective of retinex model is carring out image reconstruction, making the image after reconstruction the same as the observer saw the images at the scene. Retinex balance three aspects in compress the dynamic range of gray-scale, edge enhancement and color constancy, which can be use with different types of images and self-adaptive enhance. Retinex basic principles are to be divided into an brightness image and reflect|\}ion image, then enhance images to achieve the purpose by reducing the impact of image brightness on reflection image .According to Land's retinex model, an image can be defined as $S(x, y)$, is shown as fig (2) .

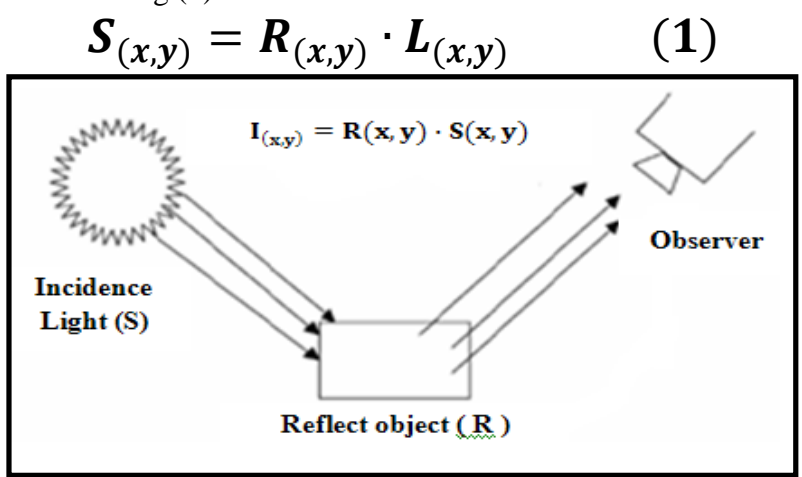

Fig 2: diagram of Retinex [7]

Where $\mathrm{L}$ expresses the brightness of the surrounding environment, and $\mathrm{R}$ is the reflectivity of objects, which includes details of the characteristics of objects. The algorithm is process of Retinex was shown in figure (3).

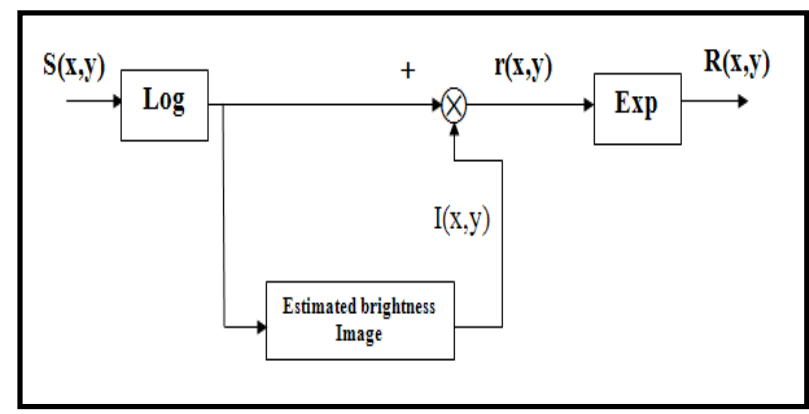

Fig 3: The algorithm Process of Retinex [7]

The Retinex Image Enhancement Algorithm is an automatic image enhancement method that enhances a digital image in terms of dynamic range compression, color independence from the spectral distribution of the scene illuminant, and color/lightness rendition. The digital image enhanced by the Retinex Image Enhancement Algorithm is much closer to the

scene perceived by the human visual system, under all kinds and levels of lighting variations, than the digital image enhanced by any other method.The multiscale retinex (MSR) is explained from single-scale retinex (SSR) [7, 8 ] :

$$
\begin{aligned}
& R_{i(x, y, c)} \\
& =\log \left[I_{i(x, y)}\right] \\
& -\log \left[F_{(x, y, c)} \otimes I_{i(x, y)}\right]
\end{aligned}
$$

Where $R_{i}(x, y, c)$ the output of channel $i(i \in \mathrm{R}, \mathrm{G}, \mathrm{B})$ at position $\mathrm{x}, \mathrm{y}$, and $\mathrm{c}$ is the Gaussian shaped surrounding space constant, $I_{i}(x, y)$ is the image value for channel $i$ and symbol $\otimes$ denoted to the convolution and $F(x, y, c)$ is the Gaussian surrounds function that is defined as $[\mathbf{9 , 1 0}]$ :

$$
F_{(x, y, c)}=k \cdot e^{\frac{-\left(x^{2}-y^{2}\right)}{c^{2}}}
$$

$\mathrm{k}$ is the normalization constant that can be determined as follows :

$$
\iint F_{(x, y, c)} d x d y=1
$$

The MSR output is then simply a weighted sum of the outputs of several different SSR output where $[\mathbf{9 , 1 0 ]}$ :

$$
\begin{gathered}
R_{M S R i}(x, y, w, c)= \\
\sum_{n=1}^{N} w_{n} R_{i}\left(x, y, c_{n}\right)(7)
\end{gathered}
$$

Where $\mathrm{N}$ is the number of scales, $\mathrm{R}_{\mathrm{i}}\left(\mathrm{x}, \mathrm{y}, \mathrm{c}_{\mathrm{n}}\right)$ the i'th component of the n'th scale, the i'th $R_{M S R i}(x, y, w, c)$ spectral component of the MSR output and the $\mathrm{w}_{\mathrm{n}}$ weight associated with the n'th scale, in which $\sum_{n=1}^{N} w_{n}=1$.

The result of the above processing will have both negative and positive RGB values, and the histogram will typically have large tails. Thus a final gain-offset is discussed in more detail below. This processing can cause image colors to go towards gray, and thus an additional processing step proposed in [9]:

$$
R^{\prime}=R_{M S R} * I_{i}^{\prime}(x, y, c)
$$

Where $\mathrm{I}_{\mathrm{i}}^{\prime}$ is given by the following formula [9]

$$
\begin{aligned}
& I_{i}^{\prime}(x, y, a, b) \\
& =b \log [1 \\
& \left.+a \frac{I_{i(x, y)}}{\sum_{i=1}^{3} I_{i(x, y)}}\right]
\end{aligned}
$$

Where the liberty to use $\log (1+x)$ was toke in place of $\log (\mathrm{x})$ to ensure a positive result. In a value of 125 is suggested for (a); for empirically settled on a value of $(b=$ 100) for a specific test image. The difference between using these two values is small. In formula (9) a second constant is used which is simply a multiplier of the result, and the final step is gain-offset by 0.35 and 0.56 respectively. The present research uses:

$(\mathrm{w} 1=\mathrm{w} 2=\mathrm{w} 3=1 / 3)$ and $(\mathrm{c} 1=250, \mathrm{c} 2=120, \mathrm{c} 3=80)$ [9].

\section{GAMMA CHARACTERISTICS CAMERA}

The output signal level characteristics with respect to the light received by the TV camera imaging element is called the camera's gamma characteristic. The output signal level that corresponds to the brightness (optical power) of the incident light, $\mathrm{P}$, is approximated by the following relation [11]: 


$$
\boldsymbol{I}=\boldsymbol{K} \boldsymbol{P}^{\gamma}
$$

The gamma $(\gamma)$ value indicates the degree of nonlinearity, $\gamma=1$ means that the output signal level is proportional to the incident light. When the TV camera is used for surveillance or other such purposes, $\gamma$ value of about 0.7 is suitable $[11,12]$.

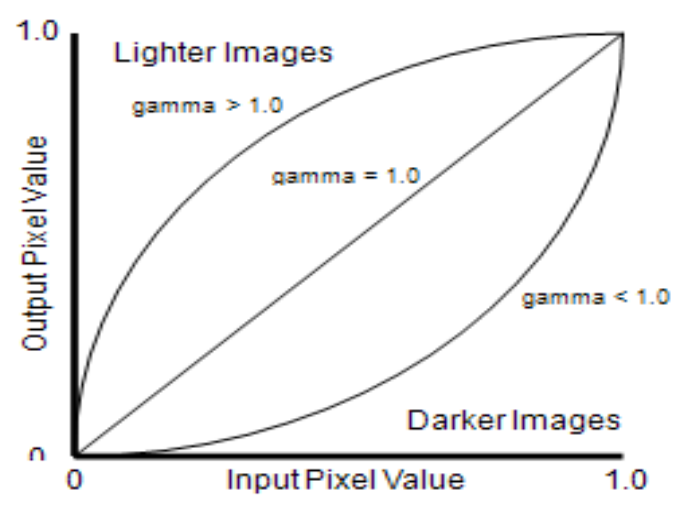

Fig 4: Show gamma less than one and gamma greater than one

In Figure (4), the pixel values range from 0.0 representing pure black, to 1.0, which represents pure white. As the figure shows gamma values of less than 1.0 darken an image. Gamma values greater than 1.0 lighten an image and a gamma equal to 1.0 produces no effect on an image. Some cameras allow switching of the $\gamma$ value. Most cameras that allow the $\gamma$ value to be selected give you the two choices of $\gamma=1$ or $\gamma=$ 0.45 .

\section{ABSOLUTE CENTRAL MOMENTUM (ACM)}

The absolute central momentum represents the statistical criteria to determine the image quality. The ACM calculates depending on the probability distribution of gray intensity as in the following relationship [13].

$$
A C M=\sum_{g=0}^{L-1}|g-\mu| P(g)
$$

where $\mathrm{P}(\mathrm{g})$ :- Probability distribution of intensity in image .
$\mathrm{g}:-$ The intensity values of image elements.
L: - Number of levels of intensity in image.

\section{THE IMAGE CONTRAST (ct)}

In this research the statistical properties of the image edge points is adopted to compute the variance by contrast equation [11], where first Imin and Imax depending on the mean of edge points $\left(\mu_{\mathrm{e}}\right)$ and the standard deviation of the edge points $\left(\sigma_{\mathrm{e}}\right)$ were calculated by following two relationships:

$$
\begin{gathered}
\mathbf{I}_{\min }=\mu_{e}-\sigma_{e}, \quad \mathrm{I}_{\max }=\mu_{e}+\sigma_{e} \\
C t=\frac{I_{\max }-I_{\text {min }}}{I_{\text {max }}+I_{\text {min }}}
\end{gathered}
$$

\section{EXPERIMENTAL DESIGN}

Figure 5 shows lighting system, which consists of a dark box painted inside with black color. The distance between test image desired for imaging and the light source is $120 \mathrm{~cm}$. The dark box includes light source (tungsten bulb) in one of its corners. The same corner at the bottom of light source has an opening for imaging to put the camera on it. On the other side the object is placed to be captured at different lighting conditions whereas lighting intensity is controlled using electronic circuit.

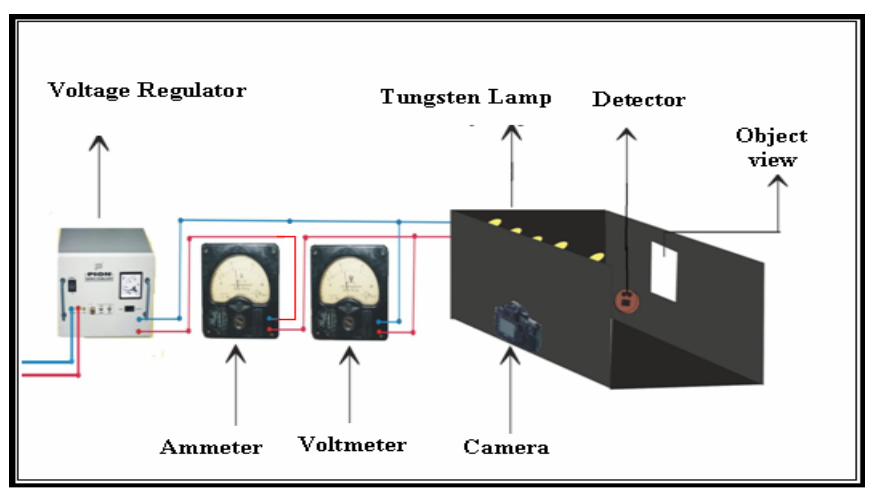

Fig 5: The Lighting System (Tungsten Light Source)

All images in this study are captured by Sony Digital Camera with different lighting intensities by the voltage applied on the light source. 12 images were captured, using a homogeneous lighting with different cases lighting, figure 6 shows the resulting images for different cases of lighting before applying enhancement method. 


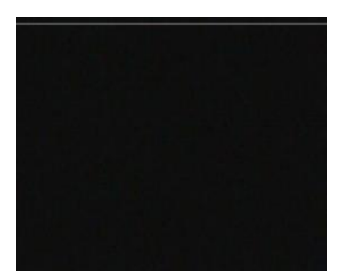

$\mathrm{V}=\mathbf{0}$

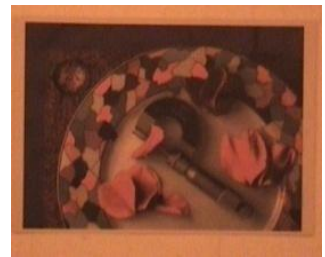

$V=120$

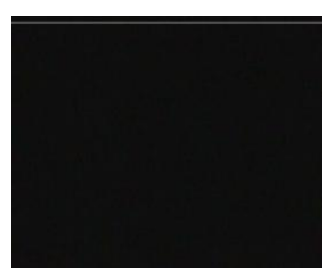

$\mathrm{V}=\mathbf{4 0}$

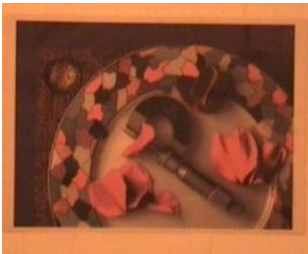

$V=140$

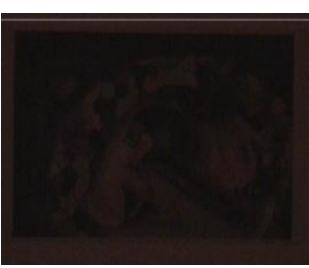

$\mathrm{V}=60$

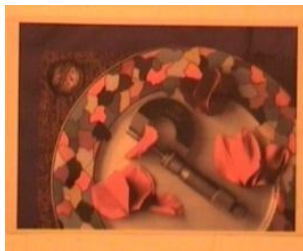

$V=160$

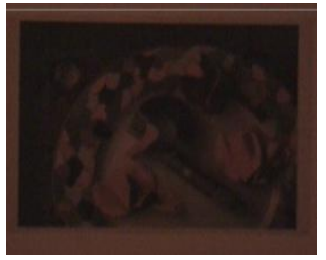

$\mathrm{V}=\mathbf{8 0}$

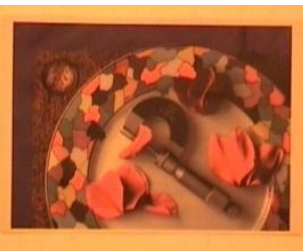

$\mathrm{V}=180$

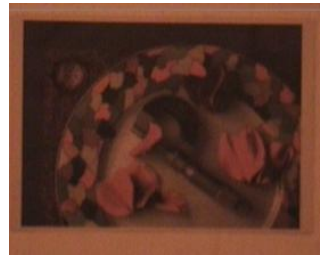

$\mathrm{V}=\mathbf{1 0 0}$

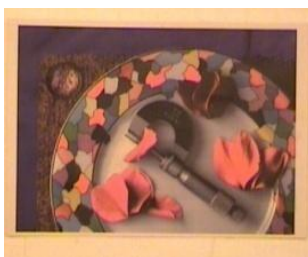

$V=200$

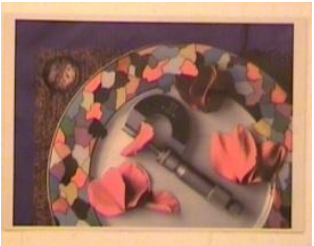

$\mathrm{V}=\mathbf{2 2 0}$

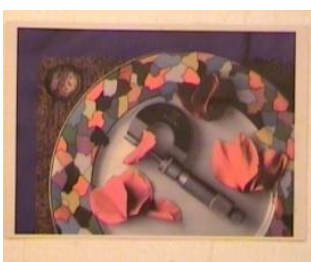

$V=240$

Fig 6: The Origin Images Using Tungsten Light Source before Using Enhancement Techniques

The other case 15 images were captured, using different lighting intensities depending on the sunlight at a different time $(6: 23 \mathrm{am}-6: 00 \mathrm{pm})$ on Sunday, Saturday on $9-2-$
2013, and figure $(7,8)$ shows the lighting system and the resulting images.

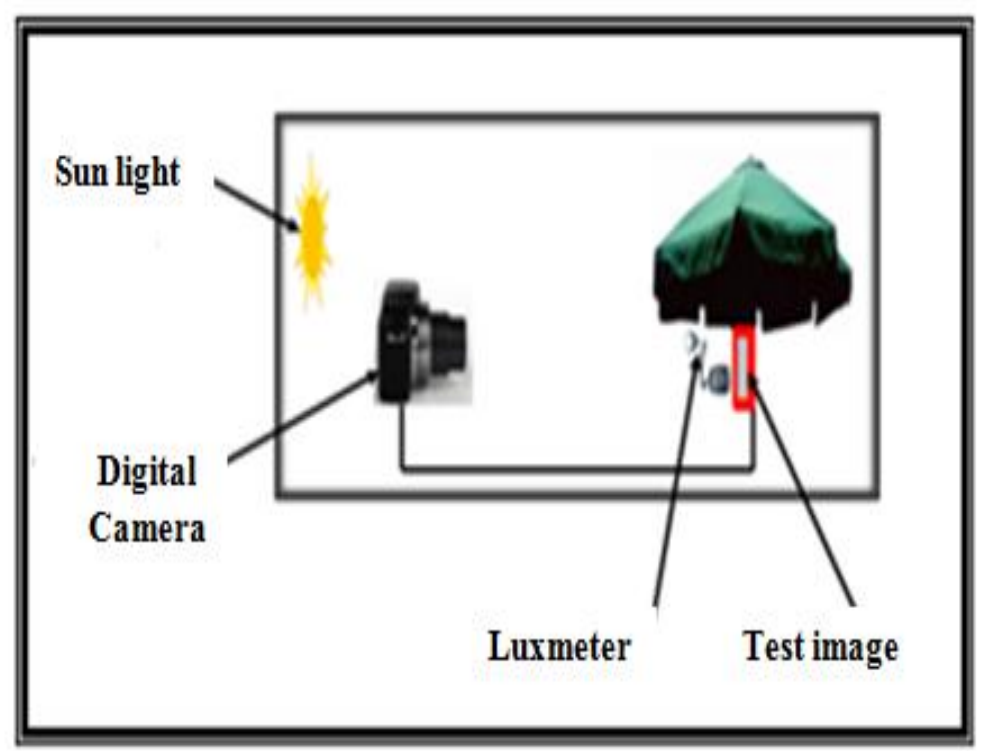

Fig 7: The Lighting System (Sun Light Source) 


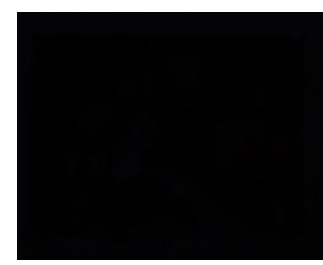

$\operatorname{lux}=0$

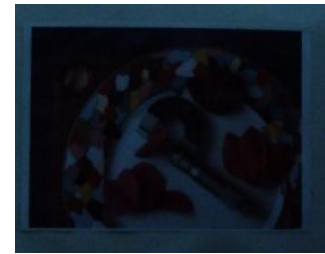

Lux=4.9

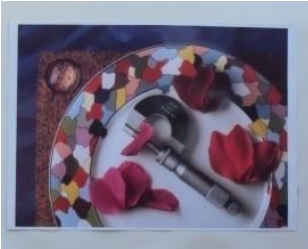

lux $=4970$

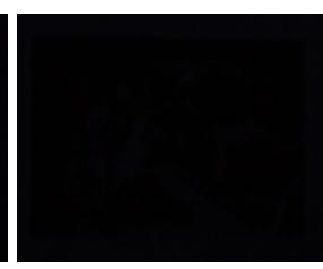

$\operatorname{lux}=0$

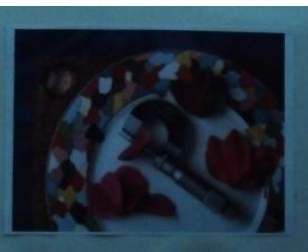

lux $=9$

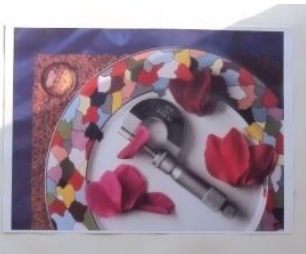

$\operatorname{lux}=9900$

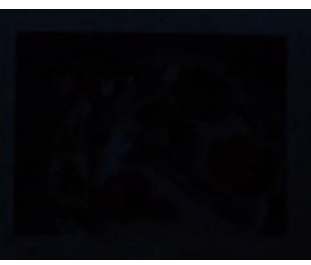

$\operatorname{lux}=1.4$

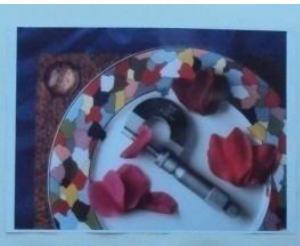

$\operatorname{lux}=29$

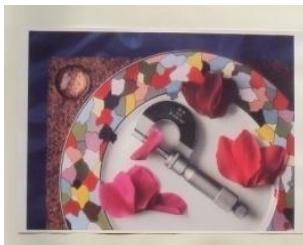

$\operatorname{lux}=\mathbf{2 1 4 0 0}$

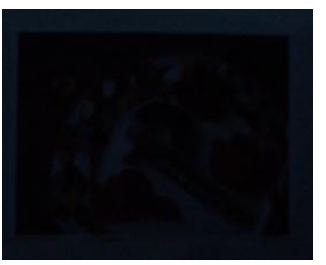

$l u x=2.3$

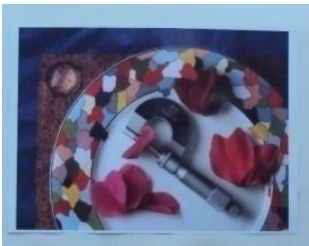

$\operatorname{lux}=131.5$

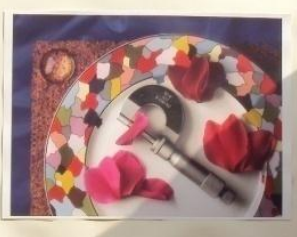

$l u x=402000$

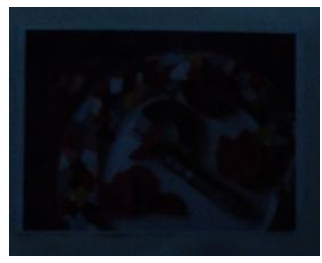

lux $=3.3$

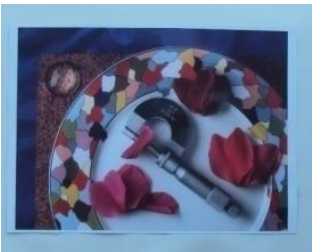

$\operatorname{lux}=599$

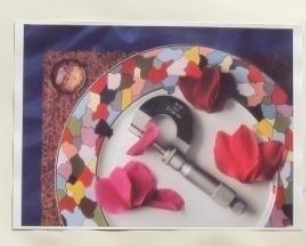

lux $=512000$

Fig 8: The Origin Images Using Sun Light Source before Using Enhancement Techniques

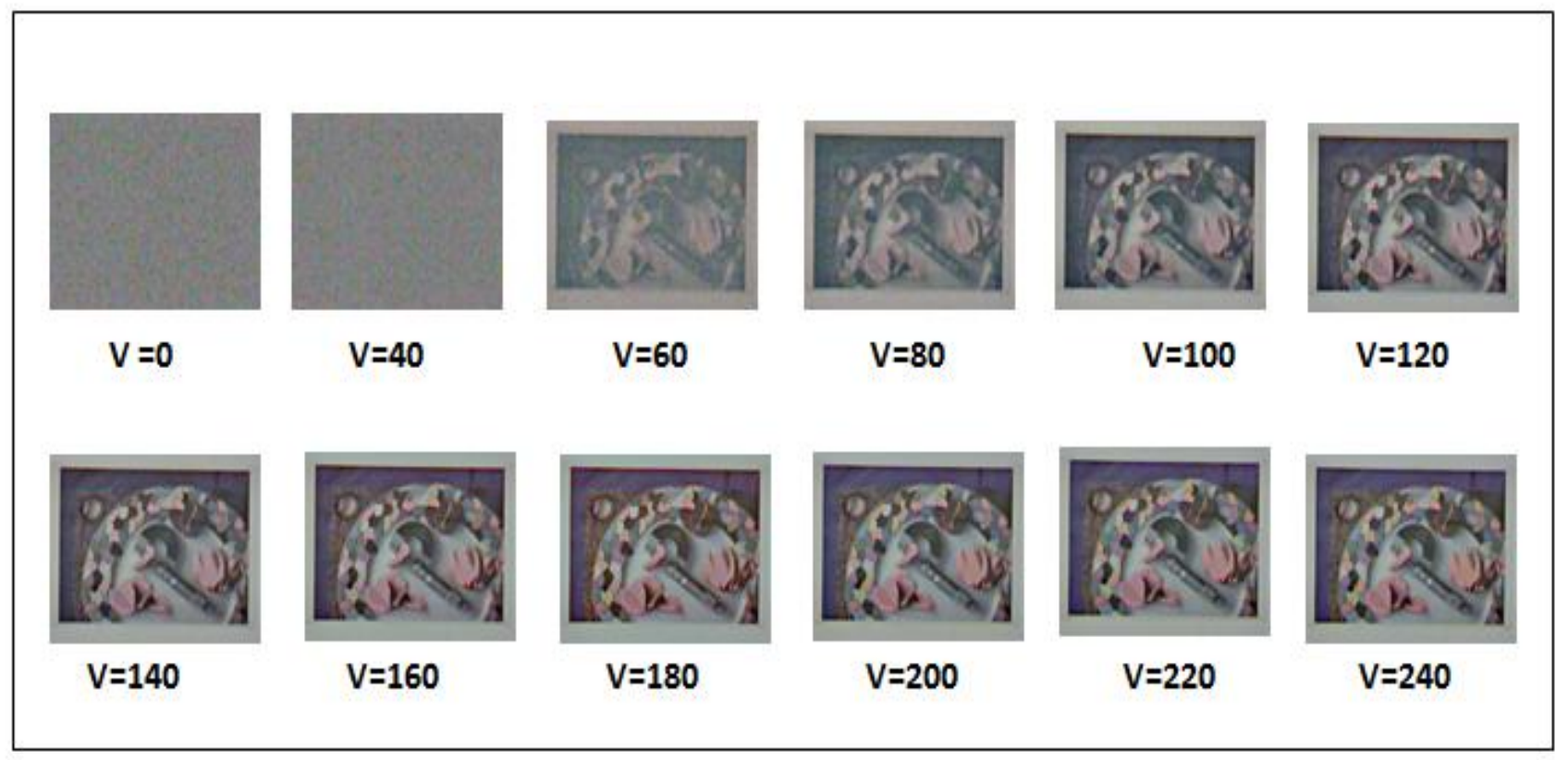

Figure 9: The Enhancement Images Obtained by Tungsten Light Source Using Retinex Technique 


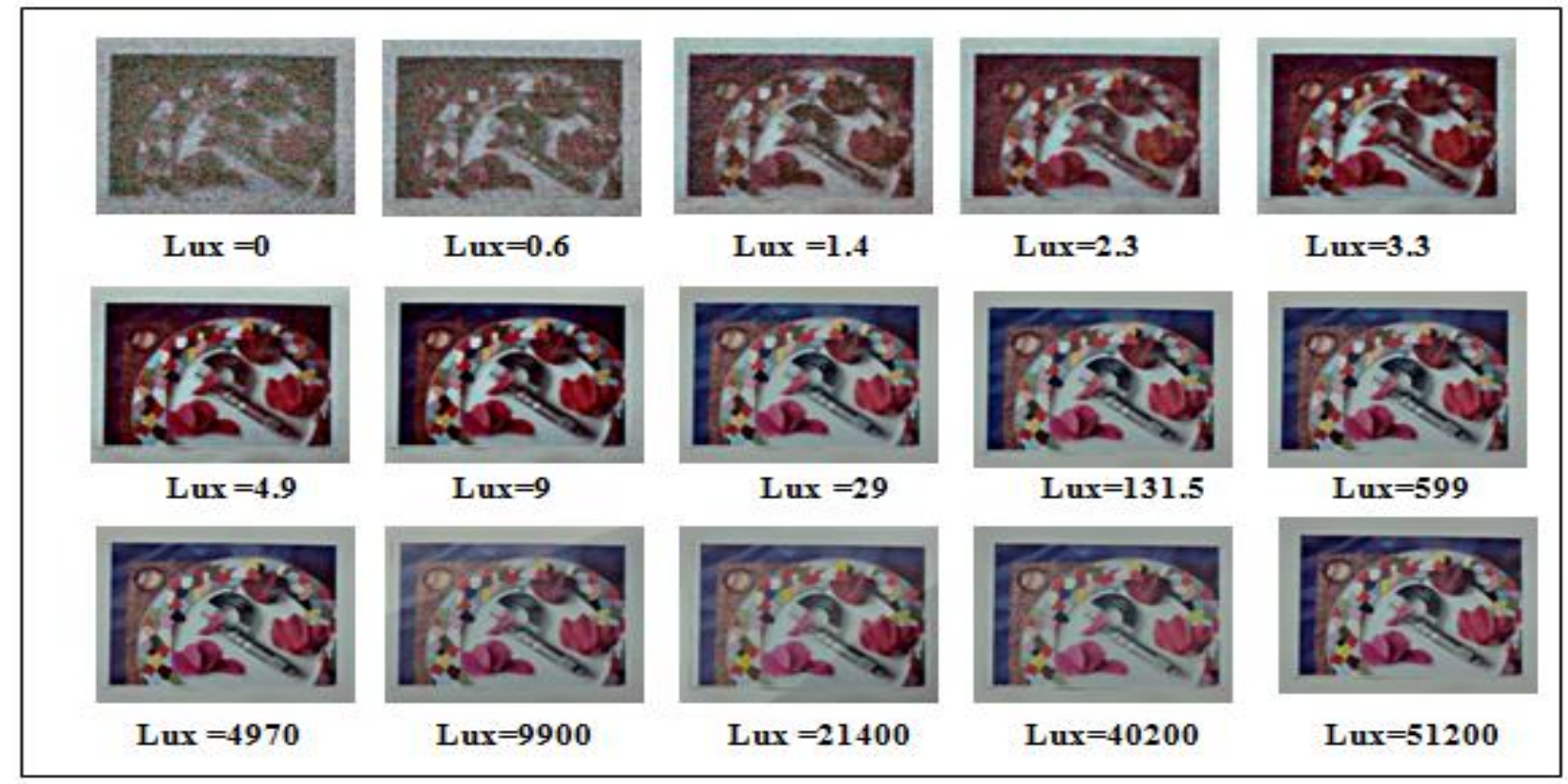

Fig 10: The Enhancement Images Obtained by Sun Light Source Using Retinex Technique

\section{RESULTS AND DISCUSION}

In this research 12 images were captured, using tungsten bulb and 15 images were captured, using sunlight at a different time. The statistical properties of each image were calculated mean, STD, ACM, and mean _contrast. The statistical properties were drawn as a function of voltage and light intensity for two lighting sources before and after using enhancement technique by Retnix, and the results can be shown in figurers (11 and 12). 


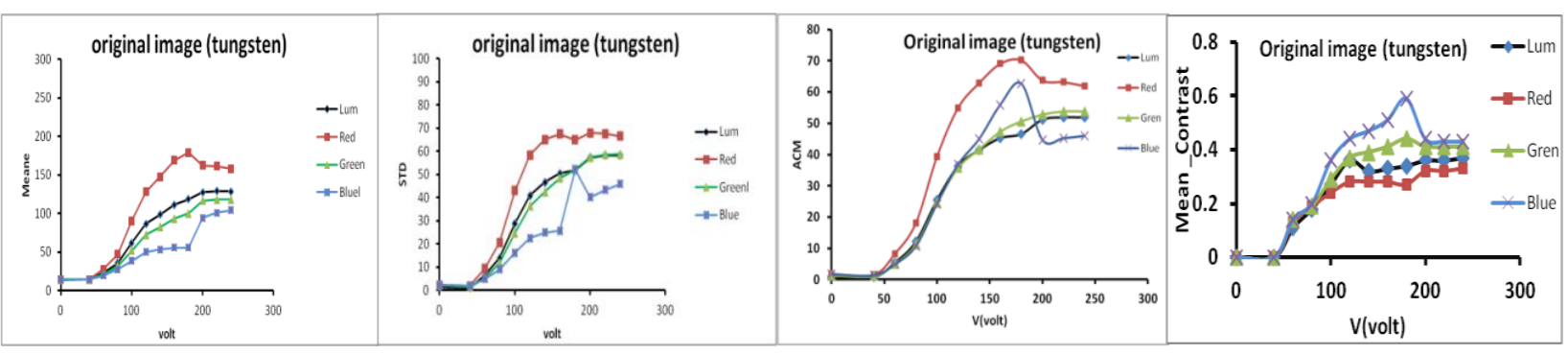

A

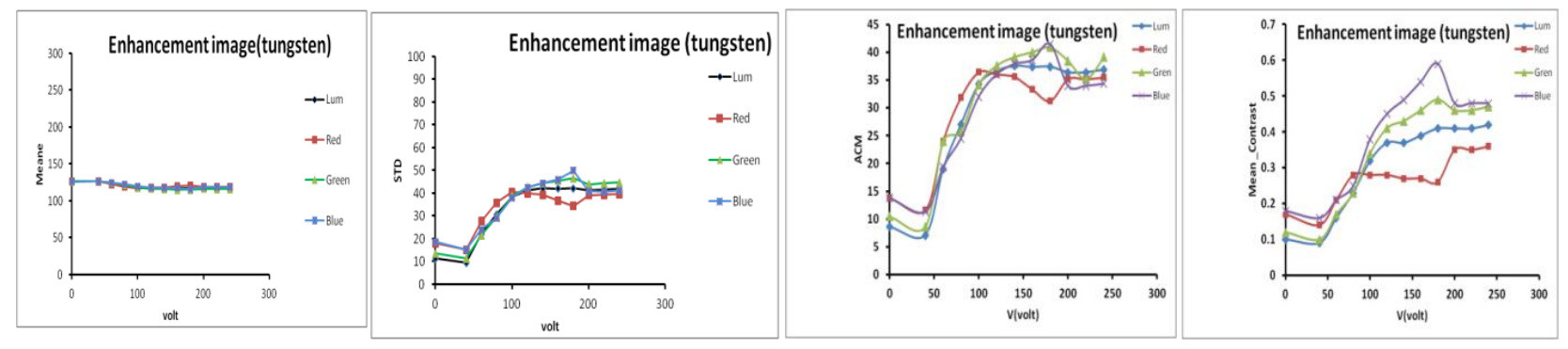

B

Fig 11: A, B The Statistical Properties as a Function of Voltage for Tungsten Lighting Sources Images before and after Using Retinex Enhancement Method Respectively.
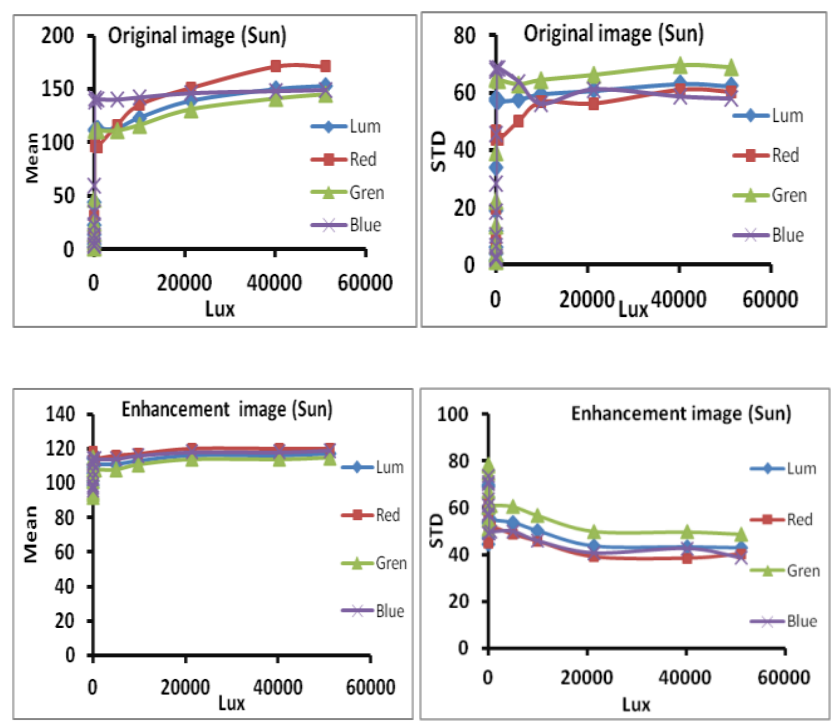

B
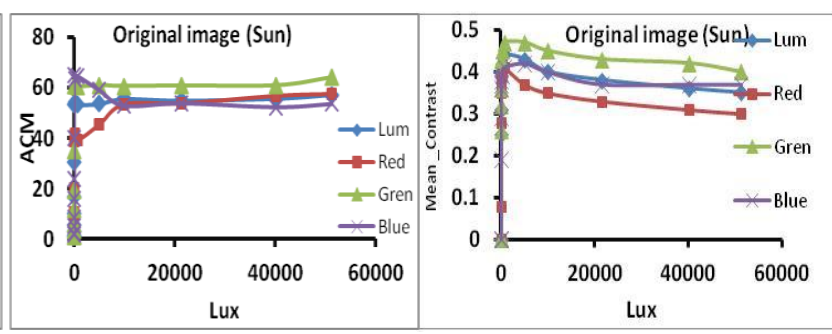

A

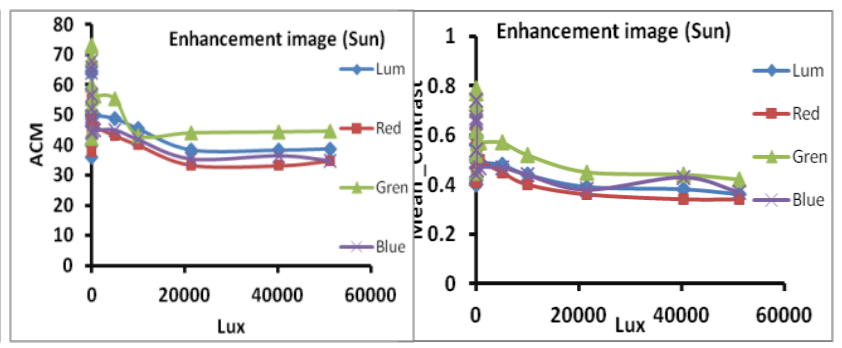

Fig 12: A, B The Statistical Properties as a Function of Light Intensity for Sun Lighting Sources Images before and after Using Retinex Enhancement Method Respectively. 
In case the images were captured, using tungsten bulb and Sun light, as clear from figure $11 \mathrm{~A}$ and $12 \mathrm{~A}$, the best image improve before enhancement methods in $\mathrm{v}=140$ volt, and the Luminous Intensity $=393 \mathrm{Lux}$ (in the medal of the day), where the value of the overall contrast are higher in this case, and when increasing the voltage and the Luminous Intensity more, the value of the contrast was decreased although increasing the light intensity. But after the enhancement process, the mean value almost equal for all enhanced images as shown in figure $11 \mathrm{~B}$ and $12 \mathrm{~B}$.

Figure 13shows the relationship between the voltages and Luminous Intensity unit of (Lux) the imaging system using optical source (lamp tungsten). From the figure the relationship between (voltage \& Luminous Intensity) is the quadratic equation was used polynomial method to find fitting the curve.

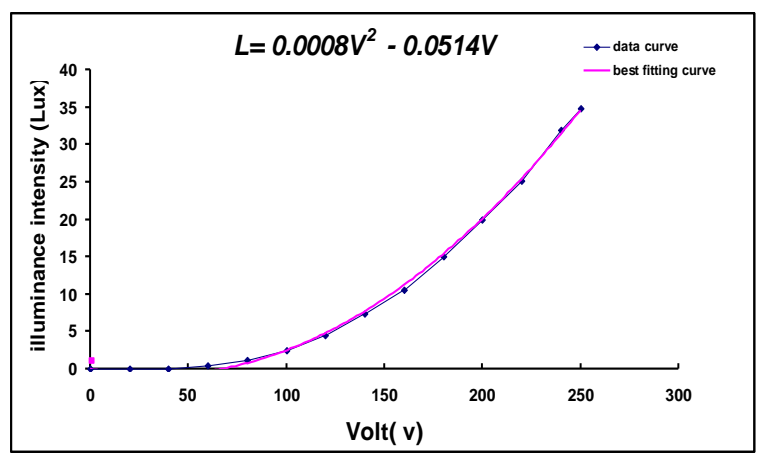

Fig 13: The relationship between the voltages and Luminous Intensity.

\section{CONCLUSION}

Retinex is an effective algorithm for source sun, as compared with other image which capture by using source tungsten. The result show that the algorithm deal with images color with better results, and improves the image mean contrast ( depend on edge of image, and the statistical properties $(\mu, \sigma$, contrast ) for images enhancement are to maintain the general attributes of statistical properties of image with different lightings according the lighting intensity of imaging system .

\section{REFERENCES}

[1] Billmeyer, Fred. W. Jr., and Max Saltzman, editors, 1981, Principles Of Color Technology, 2nd ed. New York, NY: John Wiley \& Sons.

[2] Illuminating Engineering Society of North America. 2000. IESNA Lighting Handbook: Reference \& Application, 9th ed. New York, NY: Edited by Mark S. Rea.

[3] Leslie, Russell P., and Kathryn M. Conway. 1993. The Lighting Pattern Book For Homes. Troy, NY: 4-Lighting ReseRyer, Alex. 1997. Light Measurement Handbook.

[4] Alma E.F.Taylor ,2000, illumination fundamentals ,Rensselaer Polytechnic institute

[5] Violeta Bogdanova 2010," Image Enhancement Using Retinex Algorithms and Epitomic Representation. Bulgarian Academy of Sciences Cybernetics, and Information Technologies", Volume 10, No. 3, Sofia.

[6] zhixi Bian and yan zhang ,2002, Retinex Image enhancement Techniques -algorithm ,application and advantages , Prepared in EE264 final project report .

[7] Yaoyu cheng, Yu Wang, Yan $\mathrm{Hu}, 2009$, issue 7, volume 8 ,Image enhancement algorithm based on Retinex for Small-bore steel tube butt weld's X-ray imaging , E-Mail: chengyaoyu66@163.com http://www.nuc.edu.cn/.

[8] Zia-ur Rahman, Daniel J. Jobs on, and Glenn A. Woodell.,Jonuary 2004 Retinex processing for automatic imageenhancement, Journal of Electronic Imaging.

[9] Y.Y. Schechner, J. Shamir, and N. Kiryati. Polari zation and statistical analysis of scenes containing a semireflector. J. Opt. Soc. Amer. A, 17:276-284, 2000.

[10] E. Namer and Y. Y. Schechner. In Proc. SPIE 5888, pages 36-45, 2005 Advanced visibility improvement based on polarization filtered images.

[11] John C. , " Image Processing Hand Book " , 5th edition, Materials Science and Engineering Department, North Carolina State University, (2006).

[12] Rafal C. , Richared E. , " Digital Image Processing using Matlab ", Parson Prentice - Hall , (2004) .

[13] Yaoyu cheng, Yu Wang, Yan Hu, 2009 , issue 7 , volume 8 ,"Image enhancement algorithm based on Retinex for Small-bore steel tube butt weld's X-ray imaging” , E-Mail: chengyaoyu66@163.com http://www.nuc.edu.cn/. 